\title{
The Origin of Unidentified Infrared Emission Features in Galaxies
}

\author{
Sun Kwok \\ Faculty of Science, The University of Hong Kong, Pokfulam Road, Hong Kong, China \\ email: sunkwok@hku.hk
}

\begin{abstract}
The family of unidentified infrared emission features, consisting of discrete and plateau features in the mid-infrared, are now observed in distant galaxies. A significant fraction of the total energy output of some infrared galaxies is emitted in these features. Comparisons of these features with those observed in the circumstellar and interstellar media suggest that these organic species are synthesized and ejected by evolved stars. Models of possible chemical structures of the carrier of these features are discussed.
\end{abstract}

Keywords. dust, extinction - planetary nebulae: general - ISM: lines and bands - ISM: molecules

Observations from the Spitzer Space Telescope have shown that the family of unidentified infrared emission (UIE) features are prominently present in many starburst galaxies (Smith et al. 2007). The features are so strong that it is estimated over $10 \%$ of the total energy of the galaxies are emitted in these features. These results suggest that the carriers responsible for the emission of the UIE features must be abundantly present in galaxies. Some of the UIE features, such as the 3.3, 6.2, 7.7 and $11.3 \mu \mathrm{m}$ features, have been identified as arising from aromatic compounds. Since UIE features are seen in galaxies with $z>2$, this suggests that complex organics are already present in the early Universe (Kwok 2011).

The two main questions that remain unanswered are (i) what is the chemical structure of the carrier of the UIE features; and (ii) from where are these carriers synthesized? Although the UIE carriers are popularly assumed to be PAH molecules in the astronomical community, recent work has shown that the observational properties of UIE features are more consistent with them originating from mixed aromatic/aliphatic organic nanoparticles (MAON, Kwok \& Zhang 2011). The aliphatic component is manifested in the 3.4 and $6.9 \mu \mathrm{m}$ features, as well as in strong plateau emissions around 8 and $12 \mu \mathrm{m}$.

While the UIE features are widely observed in the diffuse ISM of the Milky Way galaxy, the only definitely known site of formation of the UIE features are evolved stars. UIE features are seen to emerge over $\sim 10^{3}$ yr time scales in the late stages of stellar evolution. They first appear in the proto-planetary nebula phase and become prominent in the planetary nebula phase (Kwok 2004). Since every year one star in the Milky Way enters the planetary nebula phase, the Galaxy is constantly being enriched by large injections of stellar organics.

\section{References}

Kwok, S. 2004, Nature, 430, 985

Kwok, S. 2011, Organic Matter in the Universe, Wiley

Kwok, S. \& Zhang, Y. 2011, Nature, 479, 80

Smith, J. D. T., Draine, B. T., Dale, D. A., et al., 2007, ApJ 656, 770 